\title{
THE THIRTEENTH MARCEL GROSSMANN MEETING
}

On Recent Developments in Theoretical and Experimental General Relativity, Astrophysics and Relativistic Field Theories 


\title{
THE THIRTEENTH
}

\section{MARCEL GROSSMANN MEETING}

\section{On Recent Developments in Theoretical and Experimental General Relativity, Astrophysics and Relativistic Field Theories}

\author{
Proceedings of the MG13 Meeting \\ on General Relativity \\ Stockholm University, Stockholm, Sweden 1-7 July 2012
}

Editors

Robert T Jantzen

Villanova University, USA

Kjell Rosquist

Stockholm University, Sweden

Series Editor

Remo Ruffini

International Center for Relativistic Astrophysics

Network (ICRANet), Italy

University of Rome "La Sapienza", Italy 
Published by

World Scientific Publishing Co. Pte. Ltd.

5 Toh Tuck Link, Singapore 596224

USA office: 27 Warren Street, Suite 401-402, Hackensack, NJ 07601

UK office: 57 Shelton Street, Covent Garden, London WC2H 9HE

\section{Library of Congress Cataloging-in-Publication Data}

Marcel Grossmann Meeting on General Relativity (13th : 2012 : Stockholms universitet)

The Thirteenth Marcel Grossmann Meeting on Recent Developments in Theoretical and Experimental

General Relativity, Astrophysics, and Relativistic Field Theories : proceedings of the MG13 Meeting on

General Relativity, Stockholm University, Sweden, 1-7 July 2012 / editors Kjell Rosquist (Stockholm

University, Sweden), Robert T. Jantzen (Villanova University, USA) ; series editor Remo Ruffini (International

Center for Relativistic Astrophysics Network (ICRANet), Italy \& University of Rome "La Sapienza", Italy).

pages $\mathrm{cm}$

Includes bibliographical references and index.

ISBN 978-9814612142 (set : alk. paper) -- ISBN 978-9814612180 (v. 1 : alk. paper) --

ISBN 978-9814612197 (v. 2 : alk. paper) -- ISBN 978-9814612203 (v. 3 : alk. paper)

1. General relativity (Physics)--Congresses. 2. Gravitation--Congresses. 3. Quantum gravity--Congresses.

4. Cosmology--Congresses. I. Rosquist, Kjell, 1948- ～editor. II. Jantzen, Robert T., editor.

III. Ruffini, Remo. IV. Title. V. Title: Proceedings of the MG13 Meeting on General Relativity.

QC173.6.M37 2012

$530.11--d c 23$

2014030754

\section{British Library Cataloguing-in-Publication Data}

A catalogue record for this book is available from the British Library.

Copyright (C) 2015 by World Scientific Publishing Co. Pte. Ltd.

All rights reserved. This book, or parts thereof, may not be reproduced in any form or by any means, electronic or mechanical, including photocopying, recording or any information storage and retrieval system now known or to be invented, without written permission from the Publisher.

For photocopying of material in this volume, please pay a copying fee through the Copyright Clearance Center, Inc., 222 Rosewood Drive, Danvers, MA 01923, USA. In this case permission to photocopy is not required from the publisher.

In-house Editor: $\mathrm{Ng}$ Kah Fee 


\section{CONTENTS}

Publications in this Series . . . . . . . . . . . . . . . . . . . . . v

Sponsors . . . . . . . . . . . . . . . . . . . . . . . . . . . . . . . vii

Organizing Committees and Acknowledgements . . . . . . . . . . . . . viii

Dedication . . . . . . . . . . . . . . . . . . . . . . . . . . $\mathrm{x}$

Marcel Grossmann Awards . . . . . . . . . . . . . . . . . . . . . . . xii

Preface . . . . . . . . . . . . . . . . . . . . . . . $\mathrm{xx}$

PART A

PLENARY TALKS

On the Cosmological Singularity

Belinski, Vladimir A. . . . . . . . . . . . . . . . . . . . . . . . . . 3

GRB Afterglow Discovery with Bepposax: Its Story 15 Years Later

Frontera, Filippo . . . . . . . . . . . . . . . . . . . . . . . . . . . 33

Rotation, Convection, and Core Collapse

Arnett, W. David . . . . . . . . . . . . . . . . . . . . . . . . . . . 54

Spacetime Singularities: Recent Developments

Uggla, Claes . . . . . . . . . . . . . . . . . . . . . . . . . . . . . 59

Hidden Symmetries: From BKL to Kac-Moody

Fleig, Philipp; Nicolai, Hermann . . . . . . . . . . . . . . . . . . . . 80

Recent Results in Mathematical GR

Klainerman, Sergiu . . . . . . . . . . . . . . . . . . . . . . . . . . 93

Higher Dimensional Black Holes

Reall, Harvey $S$.

Causal Dynamical Triangulations and the Search for a Theory of

Quantum Gravity

Ambjorn, Jan; Görlich, Andrzej; Jurkiewicz, Jerzy; Loll, Renate . . . . . 120

On Quantum Gravity, Asymptotic Safety, and Paramagnetic

Dominance

Nink, Andreas; Reuter, Martin . . . . . . . . . . . . . . . . . . . . 138

Perturbative Quantum Gravity as a Double Copy of Gauge Theory

and Implication for UV Properties

Bern, Zvi . . . . . . . . . . . . . . . . . . . . . . . . . . . . . 158 
xxiv

Type Ia Supernova Cosmology: Past and Future

Goobar, Ariel . . . . . . . . . . . . . . . . . . . . . . . . . . . . 16

The Energetic Universe: A Nobel Surprise

Kirshner, Robert P. . . . . . . . . . . . . . . . . . . . . . . . . . 179

Strong, Weak, Electromagnetic and Gravitational Interactions in

Neutron Stars

Rueda, Jorge A.; Ruffini, Remo . . . . . . . . . . . . . . . . . . . . 191

Gravitational-Wave Physics and Astronomy Using Ground-Based

Interferometers

Reitze, David H.; Shoemaker, David H. . . . . . . . . . . . . . . . . 210

Gamma-Ray Burst Prompt Emission

Zhang, Bing . . . . . . . . . . . . . . . . . . . . . . . . . . . . 224

Black Holes, Supernovae and Gamma Ray Bursts

Ruffini, Remo . . . . . . . . . . . . . . . . . . . . . . . . . . . 242

Precision Tests of Theories of Gravity Using Pulsars

Kramer, Michael . . . . . . . . . . . . . . . . . . . . . . . . . . 315

The Planck Mission: Recent Results, Cosmological and Funda-

mental Physics Perspectives

Mandolesi, Nazzareno; Burigana, Carlo; Gruppuso, Alessandro;

Natoli, Paolo . . . . . . . . . . . . . . . . . . . . . . . . . . . . 333

Observation of a New Boson at a Mass of $125 \mathrm{GeV}$ with the CMS

Experiment at the LHC

Mariotti, Chiara . . . . . . . . . . . . . . . . . . . . . . 352

Unavoidable CMB Spectral Features and Blackbody Photosphere

of Our Universe

Sunyaev, Rashid A.; Khatri, Rishi . . . . . . . . . . . . . . . . . . . 373

Search for the Standard Model Higgs Boson with the ATLAS

Detector

Orestano, Domizia . . . . . . . . . . . . . . . . . . . . . . . . . 398

\section{REVIEW ARTICLES}

From the Tian San to the Tian Kong: Personal Reflections on

Fang Lizhi

Ruffini, Remo

Marcel Grossmann and his Contribution to the General Theory of

Relativity

Sauer, Tilman 
Dark Matter Particle Investigations

Bernabei, Rita . . . . . . . . . . . . . . . . . . . . . . . . . . 504

Numerical Relativity in Higher Dimensions

Sperhake, Ulrich . . . . . . . . . . . . . . . . . . . . . . . . . . 524

Nonlinear Massive Gravity and Cosmology

Mukohyama, Shinji . . . . . . . . . . . . . . . . . . . . . . . . . 548

Black Holes in Higher Dimensions (Black Strings and Black Rings)

Kunz, Jutta . . . . . . . . . . . . . . . . . . . . . . . . . . . . 568

Magneto-Plasma Processes in Relativistic Astrophysics: Modern

Developments

Tsupko, Oleg Yu. . . . . . . . . . . . . . . . . . . . . . . . . . . 582

Quantization of Entropy Spectra of Black Holes

Kwon, Yongjoon; Nam, Soonkeon . . . . . . . . . . . . . . . . . . 592

Recent Developments in Astrophysical and Cosmological Exploitation of Microwave Surveys

Burigana, Carlo; Davies, Rodney D.; de Bernardis, Paolo; et al. . . . . . 610

Features in the CMB Spectrum as an Evidence of Heavy Physics

Saito, Ryo . . . . . . . . . . . . . . . . . . . . . . . . . . . . . 648

Dark Energy

Polarski, David . . . . . . . . . . . . . . . . . . . . . . . . . . . 663

Quantum Cosmology: SUSY's Stage

Vargas Moniz, Paulo . . . . . . . . . . . . . . . . . . . . . . . . 676

The Failures of the Standard Model of Cosmology Require a New

Paradigm

Kroupa, Pavel; Pawlowski, Marcel; Milgrom, Mordehai . . . . . . . . . . 696

Physics of Non-Dissipative Ultrarelativistic Photospheres

Vereshchagin, Gregory . . . . . . . . . . . . . . . . . . . . . . . . 708

Structure of Infinity in Cosmology

Cotsakis, Spiros. . . . . . . . . . . . . . . . . . . . . . 729

Underground Gravitational Wave Observatories: KAGRA and ET

Punturo, Michele; Somiya, Kentaro . . . . . . . . . . . . . . . . . . 747

Measuring Cosmological Parameters with Gamma-Ray Bursts

Amati, Lorenzo; Della Valle, Massimo . . . . . . . . . . . . . . . . . 769

Large Scale Structure - The Seventies and Forty Years Later: From

Clusters to Clusters

Chincarini, Guido . . . . . . . . . . . . . . . . . . . . . . . . . . 781

Quantum-Gravity Phenomenology with GRB Neutrinos and

Macroscopic Bodies 
Amelino-Camelia, Giovanni . . . . . . . . . . . . . . . . . . . . 823

Symmetry Breaking in Topological Quantum Gravity

Mielke, Eckehard W. . . . . . . . . . . . . . . . . . . . . . . . . 834

Low-Magnetic-Field Magnetars

Turolla, Roberto; Esposito, Paolo . . . . . . . . . . . . . . . . . . . 852

Summary of the Session, White Dwarf Pulsars and Rotating White

Dwarf Theory

Terada, Yukikatsu . . . . . . . . . . . . . . . . . . . . . . . 870

New Developments in the LAUE Project

Liccardo, V.; Frontera, F.; Virgilli, E.; Valsan, V. . . . . . . . . . . . 881

GAME: GRB and All-Sky Monitor Experiment

Amati, Lorenzo; Campana, Riccardo; Evangelista, Yuri; Feroci,

Marco; Fuschino, Fabio; Labanti, Claudio; Salvaterra, Ruben;

Stratta, Giulia; Tagliaferri, Gianpiero . . . . . . . . . . . . . . . . . 889

Holographic Geometries for Condensed Matter Applications

Keränen, V.; Thorlacius, L. . . . . . . . . . . . . . . . . . . . . . 902

Exact Inhomogeneous Models and the Drift of Light Rays Induced

by Nonsymmetric Flow of the Cosmic Medium

Krasiński, Andrzej; Bolejko, Krzysztof . . . . . . . . . . . . . . . . . 922

\section{PART B}

\section{PARALLEL SESSIONS}

\section{- Post-Newtonian and Analytic Approximations}

Chairperson: Blanchet, Luc

Observables for Bound Orbital Motion in Axially Symmetric

Space-Times

Hackmann, Eva; Läemmerzahl, Claus . . . . . . . . . . . . . . . . . 939

Triangular Solution to the General Relativistic Three-Body

Problem

Yamada, Kei; Asada, Hideki . . . . . . . . . . . . . . . . . . . . . 942

Solving Geodesic Equations for a Test Mass in a Post-Newtonian

Approximated Kerr Spacetime Analytically Exact

Hergt, Steven; Schäefer, Gerhard; Shah, Abhay . . . . . . . . . . . . . 945

Recent Progress in Spin Calculations in the Post-Newtonian

Framework and Applications

Hartung, Johannes; Steinhoff, Jan; Schäefer, Gerhard . . . . . . . . . . 948 
On the Validity of the Adiabatic Approximation in Compact Binary Inspirals

Maselli, Andrea; Gualtieri, Leonardo; Ferrari, Valeria; Pannarale,

Francesco . . . . . . . . . . . . . . . . . . . . . . . . . . . . . 951

Construction of Gauge-Invariant Variables for Linear-Order Metric

Perturbations on an Arbitrary Background Spacetime

Nakamura, Kouji . . . . . . . . . . . . . . . . . . . . . . . . . . 954

Gravitational Waveforms for Unequal Mass Black Hole Binaries

Detectable by KAGRA

Tápai, Márton; Keresztes, Zoltán; Gergely, László Árpád . . . . . . . . . 957

Influence of Cosmological Constant Magellanic Clouds Motion and other Relevant Influences

Stuchlik, Zdeněk; Schee, Jan . . . . . . . . . . . . . . . . . . . . . 960

Virial Masses of Galaxy Clusters in the Post-Newtonian Limit

Roshan, Mahmood . . . . . . . . . . . . . . . . . . . . . . . . . 963

- Interfacing Analytical and Numerical Relativity

Chairperson: Barack, Leor

Post-Newtonian Expansion of Gravitational Waves from a Particle

in Circular Orbits Around a Black Hole

Fujita, Ryuichi . . . . . . . . . . . . . . . . . . . . . . . . . . . 969

Evolution of Inspiral Orbits Around a Schwarzschild Black Hole

Warburton, Niels; Akcay, Sarp; Barack, Leor; Gair, Jonathan;

Sago, Norichica . . . . . . . . . . . . . . . . . . . . . . . . . . . 972

Second-Order Gravitational Self-Force

Pound, Adam . . . . . . . . . . . . . . . . . . . . . . . . . . . . 975

Influence of Internal Structure on the Motion of Test Bodies in

Extreme Mass Ratio Situations

Steinhoff, Jan; Puetzfeld, Dirk . . . . . . . . . . . . . . . . . . . . 978

- Numerical Simulations of Binary Black Holes

Chairperson: Laguna, Pablo

Dynamics of Charged Black Holes

Zilhão, Miguel; Cardoso, Vitor; Herdeiro, Carlos; Lehner, Luis;

Sperhake, Ulrich . . . . . . . . . . . . . . . . . . . . . . . 983

Numerical Relativistic Null-Cone Evolutions at Fourth Order

Accuracy

Reisswig, Christian; Bishop, Nigel T.; Pollney, Denis . . . . . . . . . . 986

- Numerical Analysis of Coalescing Binaries

Chairperson: Shibata, Masaru 
xxviii

Numerical Relativity Simulations of Magnetized Black Hole-

Neutron Star Mergers

Etienne, Zachariah B.; Liu, Yuk Tung; Paschalidis, Vasileios;

Shapiro, Stuart L. . . . . . . . . . . . . . . . . . . . . . . . . . . 991

Neutron Star Evolutions Using Nuclear Equations of State with a

New Execution Model

Neilsen, David; Anderson, Matthew; Sterling, Thomas; Kaiser, Hartmut . . 995

\section{- DAMA and Related Experiments}

Chairperson: Bernabei, Rita

Dark Matter Direct Detection and LHC Searches

Fornengo, Nicolao . . . . . . . . . . . . . . . . . . . . . . . . . . 1001

Results from DAMA/LIBRA and Perspectives

Belli, Pierluigi; DAMA-collaboration . . . . . . . . . . . . . . . . . 1004

Results of the CRESST-II Dark Matter Search

Scholl, Stephan; CRESST-collaboration . . . . . . . . . . . . . . . . 1007

Rare Event Searches at Canfranc: ANAIS Experiment

Amaré, Julio; Cebrián, Susana; Cuesta, Clara; García, Eduardo;

Ginestra, Carlos; Gómez, Hèctor; Martínez, María; Oliván, Miguel

Ángel; Ortigoza, Ysrael; Ortiz de Solórzano, Alfonso; Pobes,

Carlos; Puimedón, Jorge; Sarsa, María Luisa; Villar, José Ángel;

Villar, Patricia . . . . . . . . . . . . . . . . . . . . . . . 1010

- Active Galactic Nuclei at High Energies

Chairperson: Giommi, Paolo; Aharonian, Felix

A Simplified View of Blazars: Comparison with Multi-Frequency

Observations

Giommi, Paolo; Padovani, Paolo . . . . . . . . . . . . . . . . . . . 1015

Does Black Hole Spin Play a Key Role in the FSRQ/BL Lac

Dichotomy?

Mukhopadhyay, Banibrata . . . . . . . . . . . . . . . . . . . . . . 1019

Best Fits of Simultaneous Blazar Spectral Energy Distributions

Ansoldi, Stefano; Ferigo, Domenico; Mankuzhiyil, Nijil; Persic, Massimo; Rivers, Elizabeth; Rothschild, Richard; Tavecchio, Fabrizio . . . 1022

Study of TeV Variability of Mrk 421 From 3 Years of Monitoring with the Milagro Observatory

Patricelli, Barbara; González, Maria M.; Fraija, Nissim; Marinelli, Antonio 1025

High Energy Gamma Rays from Centaurus A

Sahakyan, Narek; Yang, Ruizhi; Rieger, Frank; Aharonian, Felix;

de Ona-Wilhelmi, Emma . . . . . . . . . . . . . . . . . . . . . . . 1028 


\section{- Ultra High Energy Cosmic Rays}

Chairperson: Kampert, Karl-Heinz; Cao, Zhen

Cosmic-Ray Spectrum and Composition with the IceCube Observatory

Tamburro, Alessio; Ice Cube-Collaboration . . . . . . . . . . . . . . . 1033

Cosmic-Ray Proton to Electron Ratios

Persic, Massimo; Rephaeli, Yoel . . . . . . . . . . . . . . . . . . . 1036

Results from the Pierre Auger Observatory

De Mitri, Ivan; Pietre Auger-collaboration . . . . . . . . . . . . . . . 1039

Results from the Telescope Array Experiment

Tsunesada, Yoshiki

\section{- Higher Dimensional General Relativity}

Chairperson: Reall, Harvey S.

Gravitational Field with Sources, Regular Black Holes, Quasiblack

Holes, and Analogue Black Holes

Lemos, José P.S.; Pani, Paolo . . . . . . . . . . . . . . . . . . . . 1047

Infinite Classes of Degenerate Horizon Geometries in Higher

Dimensions

Kunduri, Hari K.; Lucietti, James . . . . . . . . . . . . . . . . . . 1056

Radiation from a $D$-dimensional Collision of Shock Waves: An

Insight Allowed by the $D$ Parameter

Coelho, Flávio S.; Herdeiro, Carlos; Rebelo, Carmen; Sampaio,

Marco O.P. . . . . . . . . . . . . . . . . . . . . . . . . . . . . 1059

Conformal Symmetry and the Gregory-Laflamme Instability

Camps, Joan . . . . . . . . . . . . . . . . . . . . . . . . . . . 1062

Asymptotic Properties of the Weyl Tensor in Higher Dimensions

Godazgar, Mahdi; Reall, Harvey S.

On the Goldberg-Sachs Theorem in Five Dimensions

Ortaggio, Marcello; Pravda, Vojtěch; Pravdová, Alena; Reall, Harvey S. . . . . . . . . . . . . . . . . . . . . . . . . . . . . . 1068

Higher Dimensional Type N and III Solutions of Einstein Gravity and Quadratic Gravity

Málek, Tomáš; Pravda, Vojtěch . . . . . . . . . . . . . . . . . . . . 1071

Critical Gravity Waves

Ayon-Beato, Eloy; Giribet, Gaston; Hassaine, Mokhtar . . . . . . . . . 1074

Warped de Sitter Solutions in the Scalar-Tensor Theory

Flachi, Antonino; Minamitsuji, Masato; Uzawa, Kunihito 
Radiation from a $D$-dimensional Collision of Shock Waves: Higher

Order Formalism and Bondi Mass

Coelho, Flávio S.; Herdeiro, Carlos; Rebelo, Carmen; Sampaio,

Marco O.P.

Warped Self-Gravitating $U(1)$ Gauge Cosmic Strings in 5D

Slagter, Reinoud . . . . . . . . . . . . . . . . . . . . . . . . . . 1083

Numerical Study of Five-Dimensional Gravitational Collapses

Yamada, Yuta; Shinkai, Hisa-aki . . . . . . . . . . . . . . . . . . . 1086

Vacuum Polarisation on the Brane for a Higher Dimensional Black

Hole Spacetime

Hewitt, Matthew; Winstanley, Elizabeth . . . . . . . . . . . . . . . . 1089

No ISCOs in Charged Myers Perry Spacetimes by Measuring

Lyapunov Exponent

Pradhan, Parthapratim . . . . . . . . . . . . . . . . . . . . . . . 1092

- Extended Theories of Gravity

Chairperson: Capozziello, Salvatore

Extended Gravity: State of the Art and Perspectives

Capozziello, Salvatore; De Laurentis, Mariafelicia . . . . . . . . . . . . 1097

How to Construct the Self/Anti-Self Charge Conjugate States?

Dvoeglazov, Valeriy V. . . . . . . . . . . . . . . . . . . . . . . . . 1113

Finsler Geometric Extension of Einstein Gravity and Observer

Transformations

Pfeifer, Christian; Wohlfarth, Mattias . . . . . . . . . . . . . . . . . 1116

Absence of Scalar Hair in Scalar-Tensor Gravity

Faraoni, Valerio; Sotiriou, Thomas P. . . . . . . . . . . . . . . . . . 1119

Stable Super-Inflating Solutions in $\mathrm{f}(\mathrm{R})$-Gravity

Ivanov, Mikhail M.; Toporensky, Alexey V. . . . . . . . . . . . . . . . 1122

From Extended Gravity with Torsion-Spin Coupling to Running

Constant for Weak-Leptonic Forces

Fabbri, Luca . . . . . . . . . . . . . . . . . . . . . . . . . . . . 1125

Super-Renormalizable Gravity

Modesto, Leonardo . . . . . . . . . . . . . . . . . . . . . . . . . 1128

No Rotating Star Solutions of a Perfect Fluid in Projectable

Horava-Lifshitz Gravity

Naoki, Tsukamoto; Tomohiro, Harada . . . . . . . . . . . . . . . . . 1131

Thermodynamics of Extended Gravity Black Holes

Cognola, Guido; Sebastiani, Lorenzo; Zerbini, Sergio . . . . . . . . . . 1134 
Testing Extended Theories of Gravity: Perspectives from the Astrometric Point of View

Vecchiato, Alberto; Gai, Mario; Capozziello, Salvatore;

De Laurentis, Mariafelicia . . . . . . . . . . . . . . . . . . . . . . 1137

Can the Chameleon Mechanism Explain Cosmic Acceleration

While Satisfying Solar System Constraints?

Hees, Aurelien; Füzfa, Andre . . . . . . . . . . . . . . . . . . . . . 1140

Aspects of Two Measures Theory

Guendelman, Eduardo I.; Vasihoun, Mahary . . . . . . . . . . . . . . 1143

AdS-Maxwell BF Theory as a Model of Gravity and Bi-Gravity

Borowiec, Andrzej; Kowalski-Glikman, Jerzy; Szczachor, Michael . . . . . 1146

$n$-DBI Gravity in a Nutshell

Coelho, Flávio S.; Herdeiro, Carlos; Hirano, Shinji; Sato, Yuki . . . . . . 1149

Hybrid $f(R)$ Theories, Local Constraints, and Cosmic Speedup

Capozziello, Salvatore; Harko, Tiberiu; Koivisto, Tomi S; Lobo,

Francisco S.N.; Olmo, Gonzalo J. . . . . . . . . . . . . . . . . . . . 1152

Tomographic Representation of Quantum and Classical Cosmology

Stornaiolo, Cosimo . . . . . . . . . . . . . . . . . . . . . . . . 1155

A Metric Approach through Mass-Length Acceleration Scales

Mendoza, Sergio . . . . . . . . . . . . . . . . . . . . . . . . . 1158

From Black Hole Quantization to Universal Scaling Laws

Capozziello, Salvatore; Cristofano, Gerardo; De Laurentis,

Mariafelicia; Luongo, Orlando . . . . . . . . . . . . . . . . . . . . 1161

Extended $f\left(R, L_{m}\right)$ Theories of Gravity

Lobo, Francisco S.N.; Harko, Tiberiu . . . . . . . . . . . . . . . . . 1164

Reheating After $f(R)$ Inflation and Observational Constraints

Nishizawa, Atsushi; Motohashi, Hayato . . . . . . . . . . . . . . . . 1167

Charged Black Holes in Palatini $f(R)$ Theories

Olmo, Gonzalo. J; Rubiera-Garcia, Diego . . . . . . . . . . . . . . . 1170

- Gravitational Fields with Sources, Regular Black Holes, Quasiblack Holes, and Analog Black Holes

Chairperson: Lemos, José P.S.; Pani, Paolo

Cylindrically Symmetric Solutions of the Einstein Field Equations

for Elastic Matter

Brito, Irene; Mena, Filipe C.; Vaz, Estelita; Carot, Jaume . . . . . . . . 1175

Conformally Flat Cylindrically Symmetric Sources and the

Linet-Tian Spacetime 
xxxii

Brito, Irene; da Silva, Maria Fátima A.; Mena, Filipe C.; Santos,

Nilton $O$.

Inhomogeneous Charged Black Hole Solutions in Asymptotically

Anti-de Sitter Spacetime

Maeda, Kengo . . . . . . . . . . . . . . . . . . . . . . . . . . . 1180

Perturbations of Spinning Black Holes: Slow-Rotation Framework

Pani, Paolo

Black Holes and Quasiblack Holes in Einstein-Maxwell Theory

Meinel, Reinhard; Breithaupt, Martin; Liu, Yu-Chun . . . . . . . . . . 1186

Charged Polytropic Stars and Quasiblack Holes

Arbañil, José D.V.; Zanchin, Vilson T.; Lemos, José P.S. . . . . . . . . 1189

Binary Inspiral in Quadratic Gravity

Yagi, Kent . . . . . . . . . . . . . . . . . . . . . . . . . . . . . 1192

Quasiblack Holes: Properties and Carter-Penrose Diagrams

Lemos, José P.S.; Zaslavskii, Oleg B.

Multihorizon Regular Black Holes

Ansoldi, Stefano; Sindoni, Lorenzo

Scalar Black Branes with Non-AdS Asymptotics

Cadoni, Mariano

Fully Explorable Horned Particles Hiding Charge

Guendelman, Eduardo; Vasihoun, Mahary

New Solutions of Charged Regular Black Holes and Their Stability

Uchikata, Nami; Yoshida, Shijun; Futamase, Toshifumi

Gravitational Vacuum Condensate Stars

Mottola, Emil; Mazur, Pawel O.

Self Sustained Traversable Wormholes in Modified Gravity

Theories

Garattini, Remo; Lobo, Francisco S.N.

Quasinormal Modes of the Draining Bathtub

Oliveira, Leandro A.; Crispino, Luís C.B.; Dolan, Samuel R.

Traversable Wormholes Supported by Dark Gravity

Lobo, Francisco S.N.

Aharonov-Bohm Effect in a Rotating Acoustic Black Hole

Oliveira, Ednilton S.; Crispino, Luís C.B.; Dolan, Samuel R. . . . . . . . 1222

On the Relation of Von Mises Equation with Acoustic Black Holes

Cherubini, Christian; Filippi, Simonetta 
Eddington Inspired Born Infeld Theory: A New Look to the Matter-Coupling Paradigm

Delsate, Terence; Steinhoff, Jan . . . . . . . . . . . . . . . . . . . . 1228

Imprints of Quadratic Theories of Gravity on Accreting Matter

Macedo, Caio F.B.; Crispino, Luis C.B.; Pani, Paolo; Cardoso, Vitor . . . 1231

Nonsingular Black Holes in Palatini Extensions of General

Relativity

Olmo, Gonzalo J.; Rubiera-Garcia, Diego . . . . . . . . . . . . . . . 1234

Apparent Horizons in Clifton-Mota-Barrow Inhomogeneous

Universe

Vitagliano, Vincenzo; Faraoni, Valerio; Sotiriou, Thomas P.;

Liberati, Stefano . . . . . . . . . . . . . . . . . . . . . . . . . . 1237

\section{- Modified Gravity}

Chairperson: Hassan, Fawad; Mukohyama, Shinji

Lorentz Violation in Gravity: Theory and Phenomenology

Sibiryakov, Sergey . . . . . . . . . . . . . . . . . . . . . . . . . . 1243

Einstein-Aether Cosmological Models

Coley, Alan; Sandin, Patrik; Alhulaimi, Bassemah . . . . . . . . . . . 1246

A New Way to Count Degrees of Freedom in dRGT Massive

Gravity Theory

Deffayet, Cédric; Mourad, Jihad; Zahariade, George . . . . . . . . . . . 1249

Cosmological Constraints on Ghost-Free Bigravity: Background

Dynamics and Late-Time Acceleration

Akrami, Yashar; Koivisto, Tomi S.; Sandstad, Marit

Consequences of Spontaneous Lorentz Violation in Gravity

Bluhm, Robert . . . . . . . . . . . . . . . . . . . . . . . . . . . 1255

Hawking-Moss Instanton in Nonlinear Massive Gravity

Zhang, Ying-li; Saito, Ryo; Sasaki, Misao . . . . . . . . . . . . . . . 1258

Rainbow Metric Formalism and Relative Locality

Loret, Niccolò; Barcaroli, Leonardo . . . . . . . . . . . . . . . . . . 1261

Spherically-Symmetric Polytrope Solutions in Massive Gravity

Verbin, Yosef ; Brihaye, Yves . . . . . . . . . . . . . . . . . . . . 1264

Differences and Similarities between Shape Dynamics and General

Relativity

Gomes, Henrique; Koslowski, Tim A. . . . . . . . . . . . . . . . . . 1267

Massive Gravity as a Limit of Bimetric Gravity

Martín-Moruno, Prado; Baccetti, Valentina; Visser, Matt . . . . . . . . 1270 
xxxiv

Cosmology Challenges Brane Scenarios in $\mathrm{AdS}_{5}$

Konikowska, Dominika

Cosmological Solutions in Biconnection and Bimetric Gravity

Theories

Vacaru, Sergiu I.

Bose-Einstein Condensate Dark Matter Model Tested by Galactic

Rotation Curves

Dwornik, Marek; Keresztes, Zoltán; Gergely, László Á. . . . . . . . . . . 1279

Definition of Good Tetrads for $f(T)$ Gravity

Tamanini, Nicola; Böhmer, Christian G. . . . . . . . . . . . . . . . . 1282

FLRW Cosmology in Weyl-Integrable Space-Time

Gannouji, Radouane; Nandan, Hemwati; Dadhich, Naresh . . . . . . . . 1285

Testing Gravity at Cosmic Scales with Clusters of Galaxies, the

CMB and Galaxy Clustering

Rapetti, David; Blake, Chris; Allen, Steven W.; Mantz, Adam;

Parkinson, David; Beutler, Florian . . . . . . . . . . . . . . . . . . 1288

Scalar-Tensor and Multiscalar-Tensor Cosmology in the General

Relativity Limit

Járv, Laur; Kuusk, Piret; Saal, Margus; Randla, Erik . . . . . . . . . . 1292

Black Hole Perturbation in Modified Gravity Theories

Motohashi, Hayato; Kobayashi, Tsutomu; Suyama, Teruaki . . . . . . . . 1295

Between General Relativity and Electrodynamics-Hamiltonian

Description of a Field Theory

Okotów, Andrzej; Świė̇ewski, Jedrzej;

Emergence of a Quasi-Newtonian Law of Gravitation: A Geometrical Impact Study

Plamondon, Réjean; Ouellet-Plamondon, Clauéric . . . . . . . . . . . . 1301

Vainshtein Mechanism with a Generalized Galileon Term

De Felice, Antonio; Kase, Ryotaro; Tsujikawa, Shinji . . . . . . . . . . 1304

Can $\gamma$ be More Than One in Scalar-Tensor Theories?

Minazzoli, Olivier . . . . . . . . . . . . . . . . . . . . . . . 1307

The Rise of a Tensor Instability in Eddington-Inspired Gravity

Escamilla-Rivera, Celia; Bañados, Maximo; Ferreira, Pedro G. . . . . . . 1310

Noether Symmetries and $\mathrm{f}(\mathrm{R})$ Theory of Gravity

Hussain, Ibrar; Ali, Sajid . . . . . . . . . . . . . . . . . . . . . . 1313

Oscillatory Regime in Multidimensional Gauss-Bonnet Cosmology

Chirkov, Dmitry M.; Toporensky, Alexey V. . . . . . . . . . . . . . . 1316 


\section{- Black Holes in Higher Dimensions (Black Rings and Black Strings)}

Chairperson: Kunz, Jutta

Black Rings in More Than Five Dimensions

Kleihaus, Burkhard; Kunz, Jutta; Radu, Eugen . . . . . . . . . . . . . 1321

Black Hole Instabilities and Local Penrose Inequalities

Figueras, Pau; Murata, Keiju; Reall, Harvey S. . . . . . . . . . . . . . 1324

New Solution-Generation-Techniques Using SL(2,R)-Duality And

General Black Holes

Tomizawa, Shinya; Mizoguchi, Shun'ya . . . . . . . . . . . . . . . . 1327

Charged Black Holes on the Taub-Bolt Instanton and Their

Thermodynamics

Nedkova, Petya G.; Yazadjiev, Stoytcho S. . . . . . . . . . . . . . . . 1330

Kaluza-Klein Rotating Multi-Black Hole Configurations with

Electromagnetic Field in Einstein-Maxwell-Dilaton Gravity

Yazadjiev, Stoytcho S. . . . . . . . . . . . . . . . . . . . . . . . . 1333

Rotating Black Rings on Taub-NUT

Chen, Yu; Teo, Edward . . . . . . . . . . . . . . . . . . . . . . . 1336

Chaos in Geodesic Motion around a Black Ring

Igata, Takahisa; Ishihara, Hideki; Takamori, Yohsuke . . . . . . . . . . 1339

Existence and Some Properties of Thermodynamic Black Di-Rings

Iguchi, Hideo; Mishima, Takashi . . . . . . . . . . . . . . . . . . . 1342

- Magneto-Plasma Processes in Relativistic Astrophysics

Chairperson: Bisnovatyi-Kogan, Gennady; Moiseenko, Sergej

Equilibrium Models of Strongly Magnetized Neutron Stars

Hirschmann, Eric W.

Magnetic Accretion in X-Ray Pulsars

Ikhsanov, Nazar R.; Likh, Yuri S.; Beskrovnaya, Nina G.;

Pustil'nik, Lev A.

Calculation of Thermal Conductivity Coefficients for Magnetized

Neutron Star

Glushikhina, Maria V.; Bisnovatyi-Kogan, Gennady S. . . . . . . . . . . 1353

Relativistic Particles in Magnetized Media Around Black Holes

Romero, Gustavo E.; Vieyro, Florencia L. . . . . . . . . . . . . . . . 1358

Relativistic Twisted Accretion Disc Around a Kerr Black Hole

Zhuravlev, Viacheslav V.; Ivanov, Pavel B.

- Black Holes

Chairperson: Kerr, Roy; Bekenstein, Jacob 
xxxvi

Canonical Quantization of Constrained Lagrangians and Conditional Symmetries: The Schwarzschild Case

Melas, Evangelos . . . . . . . . . . . . . . . . . . . . . . . . . . 1367

Accretion Disks Around Kerr Black Holes in Modified Gravity.

Pérez, Daniela; Romero, Gustavo Esteban; Perez Bergliaffa,

Santiago Esteban . . . . . . . . . . . . . . . . . . . . . . . . . . 1370

Imprints of Kerr Superspinars in the Profiled Spectral Lines of

Keplerian Rings and Discs

Schee, Jan; Stuchlik, Zdenek . . . . . . . . . . . . . . . . . . . . . 1373

Bosonic Absorption by Reissner-Nordström Black Holes

Crispino, Luís C.B.; Oliveira, Ednilton S.; Higuchi, Atsushi . . . . . . . 1375

Lyapunov Exponent and Extremal Black Hole

Pradhan, Parthapratim . . . . . . . . . . . . . . . . . . . . . . . 1378

Ultra-High Energy Collisions in the Superspinning Kerr Geometry

Stuchlik, Znedek; Schee, Jan . . . . . . . . . . . . . . . . . . . . . 1380

A Connection Between Quasinormal Modes and Nonuniqueness of

Charged Scalar-Tensor Black Holes

Doneva, Daniela D.; Yazadjiev, Stoytcho S.; Kokkotas, Kostas D.;

Stefanov, Ivan Zh.

Apparent Horizons for Black Holes Embedded in Cosmological

Backgrounds

Faraoni, Valerio

High-Velocity Collision of an ISCO Particle Around a Kerr Black Hole

Harada, Tomohiro; Kimura, Masashi

Velocity-of-Light Surfaces in Kerr and Extreme Kerr

Åman, Jan E.; Rúnarsson, Helgi Freyr

High-Velocity Collision of Two General Geodesic Particles Around

a Kerr Black Hole

Harada, Tomohiro; Kimura, Masashi

Dynamical Black Holes and Accretion in an Expanding Universe

Guariento, Daniel C.; Fontanini, Michele; da Silva, Alan M.;

Abdalla, Elcio

Entropy of a Black Hole from the Semiclassical Euclidean Action

Formulation

Eune, Myungseok; Kim, Wontae; Son, Edwin J.

Wiggly Strings of Radial Configuration in the Kerr Black Hole

Suzuki, Hiromi 
Perturbations of Kerr's Spacetime and Cosmic Censorship

Dotti, Gustavo; Gleiser, Reinaldo J. and Ranea-Sandoval, Ignacio F. 1407

- Black Hole Evaporation, Holographic Principle, and Entropic Gravity

Chairperson: Chen, Pisin

Black Hole Entropy and the Renormalization Group

Satz, Alejandro; Jacobson, Ted . . . . . . . . . . . . . . . . . . . . 1413

Seiberg-Witten Instability of Various Topological Black Holes

Ong, Yen Chin; Chen, Pisin . . . . . . . . . . . . . . . . . . . . . 1416

Study of Anisotropic Black Branes in Asymptotically Anti-de

Sitter

Maeda, Kengo

The Gauge/Gravity Duality in Einstein-Dilaton Theory

Kulkarni, Shailesh; Lee, Bum-Hoon; Park, Chanyong; Roychowd-

hury, Raju . . . . . . . . . . . . . . . . . . . . . . . . . . . . . 1422

Gauss-Bonnet Holographic Superconductors

Miskovic, Olivera; Aranguiz, Ligeia

Partition Function of a Black Hole

Mäkelä, Jarmo

Late-Time Expansion in the Semiclassical Theory of Hawking

Radiation

Menotti, Pietro

The Physics of the Far Future

Bondarescu, Ruxandra; Lundgren, Andrew P.; Bondarescu, Mihai

- Astrophysics from the Radio to Submillimetre: Planck Mission

Chairperson: Burigana, Carlo; Norgaard-Nielsen, Hans-Ulric

Introductory Remarks to Cosmic Background Parallel Sessions

Burigana, Carlo; de Bernardis, Paolo; Masi, Silvia; Norgaard-

Nielsen, Hans Ulrik

Zodiacal Light Emission in Cosmological Missions

Maris, Michele; Burigana, Carlo

Control, Assessment and Removal of Systematic Effects in Planck

Mennella, Aniello; Planck-Collaboration

Statistical Properties of Radio and Far-Infrared Extragalactic

Sources at mm/sub-mm Wavelengths

Toffolatti, Luigi; Planck-Collaboration

Neutrino Mass from SZ Surveys

Rephaeli, Yoel; Shimon, Meir 
Mixing of Blackbodies: Increasing our View of Inflation to 17 e-Folds with Spectral Distortions from Silk Damping Khatri, Rishi . . . . . . . . . . . . . . . . . . . . . . . . . . . . 1482

CMB Induced Polarization from Single Scattering by Clusters of Galaxies and Filaments Liu, Guo-Chin; Ramos, Elsa P.R.G.; da Silva, António J.C. . . . . . . . 1485 Possible Evidence of the M31 Halo Rotation in WMAP Data de Paolis, Francesco; Ingrosso, Gabriele; Nucita, Achille; Vetrugno, Daniele; Gurzadyan, Vahe G.; Kashin, Alexander; Khachatryan, Harutyun, G.; Mirzoyan, Sergey; Jetzer, Philippe; Qadir, Asghar . . . . . 1488

\section{- Inflation}

Chairperson: Sasaki, Misao

Antioscillons Mersini-Houghton, Laura . . . . . . . . . . . . . . . . . . . . . . 1493

Density Perturbations in Nonisentropic Models of Inflation with Viscous Effects Ramos, Rudnei O.; Andrade, Silvio D.C. . . . . . . . . . . . . . . . . 1497 Validity of the Semiclassical Approximation during the Preheating Phase of Chaotic Inflation

Anderson, Paul R.; Molina-Paris, Carmen; Sanders, Dillon H. . . . . . . 1500

The Evolution of Non-Adiabatic Pressure Perturbations during Multi-Field Inflation

Huston, Ian; Christopherson, Adam J. . . . . . . . . . . . . . . . . . 1504

Vorticity from Isocurvature in the Early Universe

Christopherson, Adam J.; Malik, Karim A. . . . . . . . . . . . . . . . 1507

Transport Techniques for Non-Gaussianity

Mulryne, David J.

Primordial Non-Gaussianity As a Signature of Pre-Inflationary Radiation Era

Das, Suratna

Enhanced Inflationary Trispectrum from a Non-Vacuum Initial State

Agullo, Ivan; Navarro-Salas, José; Parker, Leonard . . . . . . . . . . . 1516

Conditions for Observable Bi and Tri-Spectra in Two-Field Slow-

Roll Inflation

Elliston, Joseph .

The Viability of Potential-Driven Galileon Inflation

Ohashi, Junko; Tsujikawa, Shinji 
Quantifying the Behaviour of Curvature Perturbations Near Horizon Crossing Nalson, Ellie; Huston, Ian; Malik, Karim A.; Christopherson, Adam J.

\section{- Dark Energy and the Accelerating Universe}

Chairperson: Starobinsky, Alexei; Polarski, David

Discrimination Between Dark Energy Models from Recent

Observations

Tsujikawa, Shinji

A Two Measure Model of Dark Energy and Dark Matter with Inverse Quintessence Guendelman, Eduardo I.; Ansoldi, Stefano . . . . . . . . . . . . . . . 1534

Scalar Fields with Barotropic Equation of State: Quintessence Versus Phantom

Sergijenko, Olga; Novosyadlyj, Bohdan .

Cosmological Constant Problem and Equivalence Principle of Quantum Gravity

Mok, Ho-Ming

Cosmic Acceleration with Cosmological Soft Phonons

Rekier, Jeremy; Füzfa, André. . . . . . . . . . . . . . . . . . . . . 1543

Simplest Teleparallel Dark Energy Model

Gu, Je-An; Geng, Chao-Qiang; Lee, Chung-Chi . . . . . . . . . . . . . 1546

Polytropic Equilibrium Models with Non-Zero Cosmological

Constant

Merafina, Marco; Bisnovatyi-Kogan, Gennady S.; Tarasov, Sergey O. . . . 1549

Dark Energy and Dark Matter as Components of Cosmological

Term Stand for Vorticity and Shear

Nurgaliev, Ildus $S$.

Why We Need Dark Energy

Pavón, Diego; Radicella, Ninfa

Quintessence with Kaluza-Klein Type Couplings to Matter and an Isotropy-Violating Vector Field

Thorsrud, Mikjel

Parameterizing the Deceleration Parameter

Pavón, Diego; Duran, Ivan; del Campo, Sergio; Herrera, Ramón . . . . . 1564

Another Coincidence Problem for $\Lambda \mathrm{CDM}$ ?

van Oirschot, Pim; Kwan, Juliana; Lewis, Geraint F. 
Constraints from Cosmography in Various Parametrizations

Aviles, Alejandro; Gruber, Christine; Luongo, Orlando; Quevedo, Hernando 1570

High- $z$ Cosmography at a Glance

Vitagliano, Vincenzo; Xia, Jun-Qing; Liberati, Stefano; Viel, Matteo . . . 1574

Interacting Dark Energy and Transient Accelerated Expansion

Zimdahl, Winfried; Vargas, Cristofher Z.; Hipólito-Ricaldi, Wiliam S. . . . 1577

Cosmological Implications of Geometrothermodynamics

Luongo, Orlando; Quevedo, Hernando . . . . . . . . . . . . . . . . . 1580

Effect of Cosmic Backreaction on the Future Evolution of an

Accelerating Universe

Bose, Nilok; Majumdar, Archan S. . . . . . . . . . . . . . . . . . . 1583

$f(R)$ Gravity and the Equation of State of Geometric Dark Energy

Jaime, Luisa; Salgado, Marcelo O.P.; Patiño, Leonardo . . . . . . . . . 1586

Redshift-Drift as a Test for Discriminating Between Decelerating

Inhomogeneous and Accelerating Universe Models

Mishra, Priti; Célérier, Marie-Noëlle; Singh, Tejinder P. . . . . . . . . . 1590

Thermodynamics and New Holographic Dark Energy

Sharif, Muhammad; Saleem, Rabia . . . . . . . . . . . . . . . 1593

- Nonsingular Cosmology

Chairperson: Novello, Mario

The Generalized Second Law and the Emergent Universe

Pavón, Diego; del Campo, Sergio; Herrera, Ramón . . . . . . . . . . . 1599

Enhanced Tunnelling Models for Child Universe Formation

Ansoldi, Stefano; Guendelman, Eduardo I.; Shilon, Idan . . . . . . . . . 1602

Non-Singular Brans-Dicke- $\Lambda$ Cosmology

Alexeyev, Stanislov; Tretyakova, Darya A.; Shatskiy, Alexander

A.; Novikov, Igor D.

Connecting the Non-Singular Origin of the Universe, the Vacuum

Structure and the Cosmological Constant Problem

Guendelman, Eduardo I.; Labraña, Pedro . . . . . . . . . . . . . . . 1608

Einstein Universe Revisited and End of Dark Era

Nurgaliev, Ildus S. . . . . . . . . . . . . . . . . . . . . . . . . . 1611

- Quantum Cosmology and Quantum Effects in the Early Universe

Chairperson: Vargas Moniz, Paulo

Birth of the Universe from the Multiverse

Mersini-Houghton, Laura . . . . . . . . . . . . . . . . . . . . . . 1619 
Can One Observe Quantum-Gravitational Effects in the Cosmic

Microwave Background?

Krämer, Manuel . . . . . . . . . . . . . . . . . . . . . . . . . . 1622

Perturbations through a Quantum Bounce

Falciano, Felipe T.; Pinto-Neto, Nelson; Vitenti, Sandro D.P. . . . . . . 1625

The Bohmian Evolution of Primordial Perturbations

Santos, Grasiele; Pinto-Neto, Nelson; Struyve, Ward . . . . . . . . . . 1628

Bounds on Spontaneous Collapse Model of Quantum Mechanics

from Formation of CMBR and Standard Cosmology

Das, Suratna; Lochan, Kinjalk; Bassi, Angelo . . . . . . . . . . . . . . 1631

Dust Time in Quantum Cosmology

Husain, Viqar; Pawtowski, Tomasz . . . . . . . . . . . . . . . . . . 1634

Quantization of the Massless Scalar Field in De Sitter Spacetime

with Unitary Dynamics

Cortez, Jeronimo; Martín-de Blas, Daniel; Mena Marugán,

Guillermo A.; Velhinho, Jose M.

The Nucleation of the Thick Wall for Topological Inflation by

Oscillating Instanton Solutions

Lee, Bum-Hoon; Lee, Chul H.; Lee, Wonwoo . . . . . . . . . . . . . . 1640

Effect of Lee-Wick Thermodynamics in the Cosmology of the Early

Universe

Bhattacharya, Kaushik; Das, Suratna . . . . . . . . . . . . . . . . . 1643

Classical and Quantum Big Brake Cosmology for Scalar Field and

Tachyonic Models.

Kamenshchik, Alexander; Manti, Serena . . . . . . . . . . . . . . . . 1646

The Closed String Tachyon and its Relationship with the Evolution

of the Universe

Escamilla-Rivera, Celia . . . . . . . . . . . . . . . . . . . . . . . 1649

A Comprehensive Model of Dark Energy, Inflation and Black Holes

and the Arrow of Time

Biermann, Peter; Harms, Benjamin . . . . . . . . . . . . . . . . . . 1652

Inter-Universal Quantum Entanglement

Robles-Pérez, Salvador J.; González-Díaz, Pedro F. . . . . . . . . . . . 1655

- Experimental Gravitation

Chairperson: Laemmerzahl, Claus

Session Summary: Experimental Gravitation

Lämmerzahl, Claus . . . . . . . . . . . . . . . . . . . . . . . . . 1661

The MICROSCOPE Space Mission and the In-Orbit Calibration 
Plan for Its Instrument

Levy, Agnès; Touboul, Pierre; Rodrigues, Manuel; Hardy, Émilie;

Métris, Gilles; Robert, Alain . . . . . . . . . . . . . . . . . . . . . 1679

Observable Effects in a Class of Spherically Symmetric Static

Finsler Spacetimes

Läemmerzahl, Claus; Perlick, Volker; Hasse, Wolfgang . . . . . . . . . . 1682

A Physical Random Signal in Ether-Drift Experiments

Consoli, Maurizio; Pluchino, Alessandro . . . . . . . . . . . . . . . . 1685

Mini and Micro Black Holes: Is There a Possibility to Observe

Their Structure?

Izmaïlov, George N. . . . . . . . . . . . . . . . . . . . . . . . . . 1688

- Variation of Fundamental Constants

Chairperson: Flambaum, Victor

An Upper Limit to the Variation of $G$ from the White Dwarf

Cooling Sequence of NGC 6791

García-Berro, Enrique; Torres, Santiago; Lorén-Aguilar, Pablo;

Isern, Jordi; Althaus, Leandro G. . . . . . . . . . . . . . . . . . . . 1693

Forecast on the Variation of the Fine Structure Constant from

Combined CMB and Weak Lensing Measurements

Menegoni, Eloisa; Martinelli, Matteo; Melchiorri, Alessandro . . . . . . . 1696

- Compact Binaries and Strong-Field Tests of Gravity

Chairperson: Kramer, Michael

Probing Modified Gravity with Double Pulsars

Deng, Xue-Mei; Xie, Yi; Huang, Tian-Yi . . . . . . . . . . . . . . . 1701

New Tests of Local Lorentz Invariance and Local Position Invari-

ance of Gravity with Pulsars

Shao, Lijing; Wex, Norbert; Kramer, Michael . . . . . . . . . . . . . . 1704

Testing Standard Model Extension in Five Binary Pulsars

Xie, Yi . . . . . . . . . . . . . . . . . . . . . . . . . . 1707

Testing General Relativity using Gravitational Waves from Binary

Neutron Stars: Effect of Spins

Agathos, Michalis; Del Pozzo, Walter; Li, Tjonnie Guang Feng;

Van Den Broeck, Chris; Veitch, John; Vitale, Salvatore . . . . . . . . . 1710

- Self-Gravitating System

Chairperson: Merritt, David

Nature of the Diffuse Near-Infrared Emission in the Innermost

Arcsecond of the Galactic Center

Sabha, Nadeen; Eckart, Andreas; Merritt, David; Zamaninasab, Mohammad 1715 
Dynamical Friction in Cuspidal Galaxies

Arca-Sedda, Manuel; Capuzzo-Dolcetta, Roberto . . . . . . . . . . . . . 1718

Joint Evolution of Spinning Supermassive Black Holes and

Rotating Nuclei

Merritt, David; Vasiliev, Eugene . . . . . . . . . . . . . . . . . . . 1721

The Vast Polar Structure of the Milky Way and Filamentary

Accretion of Sub-Halos

Pawlowski, Marcel S.

Modeling the Production of Intergalactic Light in the Pre-Collapse

Phase of Galaxy Groups

Solanes, Jose M.; Darriba, Laura . . . . . . . . . . . . . . . . . . . 1727

Semidegenerate Self-gravitating System of Fermion as Dark Matter on Galaxies I: Universality Laws

Fraga, Bernardo M.O.; Argüelles, Carlos; Ruffini, Remo; Siutsou, Ivan . 1730

A Regular and Relativistic Einstein Cluster within the S2 Orbit

Centered in SgrA*

Argüelles, Carlos; Ruffini, Remo

\section{PART C}

\section{PARALLEL SESSIONS}

\section{- Photospheric Emission in GRBs}

Chairperson: Ryde, Felix; Vereshchagin, Gregory

Theory of Photospheric Emission in Gamma-Ray Bursts

Pe'er, Asaf.

Photon Thick and Photon Thin Relativistic Outflows and GRBs

Ruffini, Remo; Siutsou, Ivan; Vereshchagin, Gregory V.

Fermi GRB Spectra and the Crisis of the Band Model

Vianello, Giacomo

Radiative Transfer Near the Photosphere of Mildly and

Ultrarelativistic Outflows

Aksenov, Alexey G.; Ruffini, Remo; Vereshchagin, Gregory V.

GRB 090227B: The Missing Link Between the Genuine Short and

Long GRBs

Muccino, Marco; Ruffini, Remo; Bianco, Carlo Luciano; Izzo,

Luca; Penacchioni, Ana V.; Pisani, Giovanni Battista .

On the Decoupling of Photons from Relativistically Expanding

Outflows

Bégué, Damien; Siutsou, Ivan A.; Vereshchagin, Gregory V. 
xliv

- First Minutes of GRBs: Physics of Prompt Emission, Central Engine, and Progenitor

Chairperson: Zhang, Bing; Kumar, Pawan

Photospheres in Gamma-Ray Bursts: A Critical Overview

Pe'er, Asaf. . . . . . . . . . . . . . . . . . . . . . . . . . . . . 1765

The Family of the Induced Gravitational Collapse Scenario: The

Case of GRB 110709B

Penacchioni, Ana V.; Ruffini, Remo; Bianco, Carlo Luciano; Izzo,

Luca; Muccino, Marco; Pisani, Giovanni Battista . . . . . . . . . . . . 1768

Nucleosynthesis in the Gamma-Ray Burst Accretion Disks and

Associated Outflows

Banerjee, Indrani; Mukhopadhyay, Banibrata . . . . . . . . . . . . . . 1773

- Observations vs. Theory in the Swift Era

Chairperson: Campana, Sergio; Della Valle, Massimo

On the Dispersion of the Amati-Like Relations

Boçi, Sonila; Hafizi, Mimoza; Mochkovitch, Robert

GRB 111228, Analysis within the Induced Gravitational Collapse

Scenario and Association with a Supernova

Penacchioni, Ana V.; Ruffini, Remo; Bianco, Carlo Luciano; Izzo,

Luca; Muccino, Marco; Pisani, Giovanni Battista . . . . . . . . . . . . 1781

The Circumstellar Medium of Massive Stars: An Observable Link

Between Long Gamma-Ray Bursts and their Progenitors

van Marle, Allard Jan . . . . . . . . . . . . . . . . . . . . . . . . 1786

On a Novel Distance Indicator for Gamma-Ray Bursts Associated

with Supernovae

Pisani, Giovanni Battista; Izzo, Luca; Ruffini, Remo; Bianco,

Carlo Luciano; Muccino, Marco; Penacchioni, Ana V.; Rueda,

Jorge Armando; Wang, Yu . . . . . . . . . . . . . . . . . . . . . . 1789

Gamma-Ray Burst Host Galaxies

Savaglio, Sandra

- Gamma-Ray Burst: Correlations and Central Engine

Chairperson: Campana, Sergio; Della Valle, Massimo

Discovery of Black Hole Spindown in Hyper-Energetic CC-SNe

and Long GRBs

van Putten, Maurice H. P. M.

- Models for GRBs

Chairperson: Piran, Tsvi; Kobayashi, Shiho

GRB 090510, Explosion of a GRB in the Highest Circumburst

Medium Ever Inferred: A Disguised Short GRB 
Muccino, Marco; Ruffini, Remo; Bianco, Carlo Luciano; Izzo,

Luca; Penacchioni, Ana V.; Pisani, Giovanni Battista . . . . . . . . . . 1813

Deformed Lorentz Symmetry in Expanding Spacetimes and the

Fermi Telescope

Rosati, Giacomo

Stability of Mass Transfer in Eccentric Compact Binaries

Bobrick, Alexey; Davies, Melvyn B.; Church, Ross P.

- Exact Solutions in Four and Higher Dimensions: Mathematical Aspects Chairperson: Alekseev, Georgy

Source Integrals for Multipole Moments in Static Spacetimes

Gürlebeck, Norman

Constructive Proof of the Kerr-Newman Black Hole Uniqueness:

Derivation of the Full Solution from Scratch

Meinel, Reinhard; Richter, René

Integrability and Vesture for Axially Symmetric Harmonic Maps

Beheshti, Shabnam; Tahvildar-Zadeh, Shadi

On the Monodromy Transform Approach to Solution of

String Gravity and Supergravity Equations in Four and

Higher Dimensions

Alekseev, George A.

Ricci Collineations of Plane Symmetric Lorentzian Manifolds

Admitting Six Isometries

Ziad, Muhammad .

Classification of a Class of Plane Symmetric Static Spacetimes

According to the Noether Symmetries

Tooba, Feroze; Bismah, Jamil .

\section{- Cosmological Singularities and Asymptotics}

Chairperson: Cotsakis, Spiros

The Paradox of Soft Singularity Crossing Avoided by

Distributional Cosmological Quantities

Kamenshchik, Alexander; Keresztes, Zoltán; Gergely, László Á. . . . . . . 1847

Asymptotic Solution in $f(R)$-Gravity

Toporensky, Alexey; Bukzhalev, Evgeny

Can Second Order Gravity Theory Explain Acceleration?

Miritzis, John

Effective Gravity and Homogenous Solutions

Müller, Daniel 
xlvi

Dynamics and Asymptotics of Brane-Worlds

Ignatios, Antoniadis; Spiros, Cotsakis; Ifigeneia, Klaoudatou . . . . . . . 1859

Limits of Isotropic Universes with Interacting Fluids

Spiros, Cotsakis; Kittou, G. . . . . . . . . . . . . . . . . . . . . . 1862

Generic Regular Universes in Higher Order Gravity Theories

Cotsakis, Spiros; Trachilis, Dimitrios; Tsokaros, Antonios

Asymptotic States of Generalized Universes with Higher

Derivatives

Cotsakis, Spiros; Kolionis, Georgios; Tsokaros, Antonios . . . . . . . . . 1868

Series Expansions and Sudden Singularities

Barrow, John D.; Cotsakis, Spiros; Tsokaros, Antonios . . . . . . . . . 1871

\section{- Theoretical Issues in GR}

Chairperson: Brill, Dieter

A Reference for Our Covariant Hamiltonian Boundary Term

Nester, James M.; Chen, Chiang-Mei; Liu, Jian-Liang; Sun, Gang .. . 1877

Hamiltonian Dynamics in Extended Phase Space for Gravity

and Its Consistency with Lagrangian Formalism: A Generalized

Spherically Symmetric Model as an Example

Shestakova, Tatyana P.

Describing Accelerated Paths in Flat Background as a Geodesic

Motion in a Curved Metric

Bittencourt, Eduardo; Novello, Mario

Shape Dynamics and Gauge-Gravity Duality

Gomes, Henrique; Koslowski, Tim. A . . . . . . . . . . . . . . . . . 1886

Inertial Frames without the Relativity Principle: Breaking Lorentz

Symmetry

Baccetti, Valentina; Tate, Kyle; Visser, Matt . . . . . . . . . . . . . . 1889

Stability of Equatorial Circular Geodesics for Kerr-Newman

Spacetime via Lyapunov Exponent

Pradhan, Parthapratim . . . . . . . . . . . . . . . . . . . . . . . 1892

Trivial Gauge Transformations in Poincaré Gauge Gravity

Banerjee, Rabin; Roy, Debraj . . . . . . . . . . . . . . . . . . . . . 1895

Geodesic Flows and Their Deformations in Bertrand Spacetimes

Kumar, Prashant; Bhattacharya, Kaushik; Sarkar, Tapabrata . . . . . . . 1898

Vacuum Decomposition of Einstein's Theory and Knot Topology

of Vacuum Space-Time

Cho, Yongmin 
The Biconnection Variational Principle for General Relativity

Tamanini, Nicola . . . . . . . . . . . . . . . . . . . . . . . . . . 1909

A Novel Aspect of Analogue Gravity

Toniato, Júnior D.; Goulart, Érico; Novello, Mario; Falciano,

Felipe T. . . . . . . . . . . . . . . . . . . . . . . . . . . . . . . 1912

Extremal Versus Non-Extremal Blackhole

Pradhan, Parthapratim . . . . . . . . . . . . . . . . . . . . . . . 1915

Existence of Double Null Form for $(2+1)$-Dimensional Spacetimes

Siddiqui, Azad A.; Riaz, S.M. Jawwad . . . . . . . . . . . . . . . . . 1917

\section{- Exact Solutions (Physical Aspects)}

Chairperson: Scott, Susan

Properties of a Two-Sphere Singularity

Konkowski, Deborah A.; Helliwell, Thomas M. . . . . . . . . . . . . . 1923

Friction Forces in General Relativity

Bini, Donato; Gregoris, Daniele; Kjell, Rosquist . . . . . . . . . . . . 1926

Conformal Isometry of a Lukewarm Black Hole

Bengtsson, Ingemar; Rúnarsson, Helgi Freyr . . . . . . . . . . . . . . 1929

Slicing Black Hole Spacetimes

Bini, Donato; Geralico, Andrea . . . . . . . . . . . . . . . . . . . . 1932

Linearised Stability Analysis of Generic Thin Shells

Lobo, Francisco S.N.; Martín-Moruno, Prado; Garcia, Nadiezhda;

Visser, Matt . . . . . . . . . . . . . . . . . . . . . . . . . . . . 1935

Exact Gravitational Waves in Higher Dimensions

Podolský, Jiřı́; Švarc, Robert . . . . . . . . . . . . . . . . . . . . . 1938

Scattering of Particles by Radiation Fields: A Comparative

Analysis

Haney, Maria; Bini, Donato; Geralico, Andrea; Jantzen, Robert T. . . . . 1941

\section{- Quantum Fields}

Chairperson: Belinski, Vladimir

A Summary: Quantum Singularities in Static and Conformally

Static Space-times

Konkowski, Deborah A.; Helliwell, Thomas M. . . . . . . . . . . . . . 1947

Some Interesting Aspects of Hawking Radiation

Sharif, Muhammad; Javed, Wajiha

Breakdown of the Equivalence between Passive Gravitational Mass

and Energy for a Quantum Body

Lebed, Andrei G.

Thermal Aspects in Curved Metrics 
xlviii

Acquaviva, Giovanni . . . . . . . . . . . . . . . . . . . 1956

Scalar Field Hadamard Renormalisation in $\mathrm{AdS}_{\mathrm{n}}$

Kent, Carl; Winstanley, Elizabeth . . . . . . . . . . . . . . . . . 1959

Infrared Issues in Graviton Higgs Theory

Bhattacharjee, Srijit; Majumdar, Parthasarathi . . . . . . . . . . . . . 1962

Rotating Fermions

Ambrus, Victor E.; Winstanley, Elizabeth . . . . . . . . . . . . . . . 1965

Chiral Anomaly for V-A Fields in 6 Dimensional Curved Space

Yajima, Satoshi; Fukuda, Makoto; Oka, Tomonori; Eguchi, Kohe;

Yamashita, Shinji; Yamamoto, Takuo . . . . . . . . . . . . . . . . . 1968

QFT on de Sitter: Lorentzian Flat Chart and Euclidean Sphere

Korai, Yusuke; Tanaka, Takahiro . . . . . . . . . . . . . . . . . 1971

Hawking Radiation for a Proca Field: The Coupled Modes

Herdeiro, Carlos; Sampaio, Marco O.P.; Wang, Mengjie . . . . . . . . . 1974

The Phase Space Quantum Description of Linearized Gravitational

Field

García-Compeán, Hugo; Turrubiates, Francisco J. . . . . . . . . . . 1977

Vilkovisky-DeWitt Effective Potential Revisited in Gauge-Free

Framework

Bhattacharjee, Srijit. . . . . . . . . . . . . . . . . . 1981

- Sources of Gravitational Waves

Chairperson: Melatos, Andrew

Stochastic Gravitational-Wave Background

Mandic, Vuk . . . . . . . . . . . . . . . . . . . . . . . . . . . . 1985

Gravitational Waves from Core-Collapse Supernovae and

Collapsars

Kotake, Kei . . . . . . . . . . . . . . . . . . . . . . . . . . . . 1989

Mountains and R-Modes in Low-Mass X-Ray Binaries

Haskell, Brynmor . . . . . . . . . . . . . . . . . . . . . . . . . . 1993

Neutron Star Oscillations from Starquakes

Keer, Lucy. C; Jones, David Ian . . . . . . . . . . . . . . . . . . . 1996

- Gravitational Wave and Multimessenger Astronomy

Chairperson: Ricci, Fulvio; Marka, Szabolcs

Neutrino Physics and Astrophysics with the ANTARES Neutrino

Telescope

Spurio, Maurizio . . . . . . . . . . . . . . . . . . . . . . . 2001

Gravitational Wave and High Energy Neutrino Multimessenger

Searches 
Bartos, Imre . . . . . . . . . . . . . . . . . . . . . . . . . . 2004

Joint Search for Gravitational Waves and Neutrinos from

Core-Collapse Supernovae

Re, Virginia . . . . . . . . . . . . . . . . . . . . . . . 2007

The Invisible Messengers: The 2007 Data Search between

Gravitational Waves and High Energy Neutrinos

Di Palma, Irene . . . . . . . . . . . . . . . . . . . . . . . . . . 2009

Multichannel GW Burst Detection with Baksan Neutrino Obser-

vatoru Setups

Rudenko, Valentin N.; Popov, Sergei B. . . . . . . . . . . . . . . . . 2012

Search for Continuous Gravitational Waves from Known Neutron

Stars: Status and Future Prospects

Palomba, Cristiano . . . . . . . . . . . . . . . . . . . . . . 2016

Measuring the Redshift of Standard Sirens Using the Neutron Star

Deformability

Li, Tjonnie Guang Feng; Del Pozzo, Walter; Messenger, Chris . . . . . . 2019

- Status of the Gravitational Wave Detectors

Chairperson: Blair, David

Advanced Virgo: An Update

Fafone, Viviana. . . . . . . . . . . . . . . . . . . . . . . 2025

Benefit of a Larger Detector Array for Prompt Gravitational Wave

Detection

Chung, Shin Kee; Chu, Qi; Hooper, Shaun; Wen, Linqing; Liu,

Yuan; Du, Zhihui . . . . . . . . . . . . . . . . . . . . . . . . . . 2029

Measurement of Mechanical Excess Noise from the Stressed Silicate

Bonding

Pashentseva, Maria; Bilenko, Igor .

\section{- Underground Gravitational Wave Detectors}

Chairperson: Lueck, Harald; Lazzarini, Albert

Seismic Attenuation System (SAS) in the Kamioka Mine

Takahashi, Ryutaro; Yamamoto, K.; Uchiyama, T.; Sekiguchi, T.;

Ishizaki, H.; Takamori, A.; DeSalvo, R.; Majorana, E.; van den

Brand, J.; Hennes, E.; Bertolini, A. . . . . . . . . . . . . . . . . . . 2037

Squeezed-Light Lasers for Gravitational Wave Observatories

Khalaidovski, Alexander; Vahlbruch, Henning; Schnabel, Roman . . . . . 2040

- History of Relativity and Cosmology

Chairperson: Bracco, Christian; Ohanian, Hans

Poincaré and Relativity: The Logic of the 1905 Palermo Memoir 
Bracco, Christian; Provost, Jean-Pierre . . . . . . . . . . . . . . 2045

Edoardo Amaldi and the Birth of the Gravitational Wave Research in Italy

Coccia, Eugenio . . . . . . . . . . . . . . . . . . . . . 2048

Quanta: The Originality of Einstein's Approach to Relativity?

Bracco, Christian . . . . . . . . . . . . . . . . . . . . . 2051

A Brief History of the Energy-Momentum Tensor; 1900-1912.

Provost, Jean-Pierre . . . . . . . . . . . . . . . . . . . . . . . . . 2054

Weyl's Raum, Zeit, Materie and Its Early Reception

Eckes, Christophe. . . . . . . . . . . . . . . . . . 2057

Weyl's Abandonment of Unified Field Theory

Sieroka, Norman . . . . . . . . . . . . . . . . . . . . . . . . . . 2061

Transits of Venus and the Astronomical Unit: Four Centuries of Increasing Precision

Sigismondi, Costantino . . . . . . . . . . . . . . . . . . . . . . . 2064

- Supernova Cosmology and the Accelerating Universe

Chairperson: Goobar, Ariel; Sollerman, Jesper

Towards the Future of Supernova Cosmology

Knights, Michelle; Bassett, Bruce A.; Varughese, Melvin; Hlozek,

René; Kunz, Martin; Smith, Mat; Newling, James . . . . . . . . . . . . 2069

- Observational Gravitational Lensing [Microlensing]

Chairperson: Jetzer, Philippe

Microlensing in Globular Clusters: The First Confirmed Lens.

Jetzer, Philippe . . . . . . . . . . . . . . . . . . . 2075

Free-Floating Planets Towards the Euclid's Field of View

Hafizi, Mimoza; Hamolli, Lindita . . . . . . . . . . . . . . . . . . . 2079

Diffractive Microlensing: A New Probe of the Local Universe

Heyl, Jeremy S. . . . . . . . . . . . . . . . . . . . . . . . . . . . 2082

Microlensing Towards the Small Magellanic Cloud

Mirzoyan, Sergey . . . . . . . . . . . . . . . . . . . . 2085

Finding Gravitational Arcs in Wide Field Surveys: The Mediatrix

Arcfinder and Comparison to Other Methods in a Simulated

Sample

De Bom, Clecio; Furlanetto, Cristina; More, Anupreeta; Brandt,

Carlos; Makler, Martn; Santiago, Baslio . . . . . . . . . . . . . . 2088

Strong Gravitational Lensing by Phantom Black Holes

Stefanov, Ivan Zh.; Gyulchev, Galin N. . . . . . . . . . . . . . . . . 2091 
Polarization Profiles for Selected Microlensing Events Towards the Galactic Bulge

Nucita, Achille A.; Ingrosso, Gabriele; Calchi-Novati, S.; de

Paolis, Francesco; Jetzer, Philippe; Strafella, F.; Zakharov, A.F. 2094

\section{- Cosmology from GRBs}

Chairperson: Amati, Lorenzo; Yonetoku, Daisuke

High Redshift Cosmography: New Results and Implications for

Dark Energy

Demianski, Marek; Piedipalumbo, Ester; Rubano, Claudio;

Scudellaro, Paolo . . . . . . . . . . . . . . . . . . . . . . . . . . 2099

Hints for a Physically based GRB Distance Indicator

Izzo, Luca; Pisani, Giovanni Battista; Muccino, Marco; Ruffini,

Remo; Bianco, Carlo Luciano; Enderli, Maxime; Wang, Yu . . . . . . . 2102

Determination of Intrinsic Slope of the Luminosity-Time Correla-

tion in X-Ray Afterglows of GRBs and Its Implications

Dainotti, Maria . . . . . . . . . . . . . . . . . . . . . . . . . . . 2106

Searching for Tight Gamma-Ray Burst Luminosity Relations

Qi, Shi; Lu, Tan . . . . . . . . . . . . . . . . . . . . . . . . . . 2110

- New Developments in the Study of the Large Scale Structure of the Universe

Chairperson: van de Weygaert, Rien

NEXUS of the Cosmic Web

Cautun, Marius; van de Weygaert, Rien; Jones, Bernard J.T.;

Frenk, Carlos S.; Hellwing, Wojciech A.; . . . . . . . . . . . . . . . . 2115

Multimodality of Rich Clusters from the SDSS DR8 within the

Supercluster-Void Network

Einasto, Maria

Less is more: How Cosmic Voids Can Shed Light on Dark Energy

Bos, E.G. Patrick; van de Weygaert, Rien; Ruwen, Jarno; Dolag,

Klaus; Pettorino, Valeria

Evidence for Alignment of Galaxies in Filaments

Tempel, Elmo; Saar, Enn; Stoica, Radu S.

Precision Cosmology with the 2MASS Clustering Dipole

Bilicki, Maciej; Chodorowski, Michal; Hellwing, Wojciech; Jarrett,

Thomas; Mamon, Gary . . . . . . . . . . . . . . . . . . . . . . . 2127

Hierarchical Clustering and the BAO Signature

Hellwing, Wojciech A.; Juszkiewicz, Roman; van de Weygaert,

Rien; Bilicki, Maciej 
The Phase-Space Structure of Cold Dark Matter in the Universe

Shandarin, Sergei . . . . . . . . . . . . . . . . . . . . . . . . . . 2133

ORIGAMI: Delineating Cosmic Structures with Phase-Space

Folding

Neyrinck, Mark C.; Falck, Bridget L.; Szalay, Alex S. . . . . . . . . . . 2136

Tracing, Analyzing and Visualizing Dark Matter in Phase Space

Hahn, Oliver; Abel, Tom; Kaehler, Ralf . . . . . . . . . . . . . . . . 2139

Adhesion and the Geometry of the Cosmic Web

Hidding, Johan; Van de Weygaert, Rien; Vegter, Gert; Jones,

Bernard J. T. . . . . . . . . . . . . . . . . . . . . . . . . . . 2142

- Loop Quantum Gravity, Quantum Geometry, Spin Foams

Chairperson: Lewandowski, Jerzy

Loop Quantization of Shape Dynamics

Koslowski, Tim A. . . . . . . . . . . . . . . . . . . . . . . . . . 2147

Spin Foam Models as Effective Theories: Abelian Case

Zapata, José A. . . . . . . . . . . . . . . . . . . . . . . . . . . . 2150

Kerr-Schild Way to Quantum Gravity

Burinskii, Alexander . . . . . . . . . . . . . . . . . . . . . . . 2153

The Transfer Matrix in Four-Dimensional Causal Dynamical

Triangulations

Görlich, Andrzej . . . . . . . . . . . . . . . . . . . . 2156

Graph Diagrams in Transition Amplitudes of Dipole Cosmology

Puchta, Jacek; Kisielowski, Marcin; Lewandowski, Jerzy . . . . . . . . . 2159

Coupling Dimers to CDT - Conceptual Issues

Glaser, Lisa . . . . . . . . . . . . . . . . . . . . . . . . . . . . 2162

A Computable Framework for Loop Quantum Gravity

Husain, Viqar; Pawtowski, Tomasz . . . . . . . . . . . . . . . . . . 2165

Fields and Laplacians on Quantum Geometries

Thürigen, Johannes . . . . . . . . . . . . . . . . . . . . . . . . . 2168

A New Perspective on Early Cosmology

Alesci, Emanuele . . . . . . . . . . . . . . . . . . . . . . . . . . 2171

Hidden Symmetries and Gauge Theories

Ferreira, Luiz A.; Luchini, Gabriel . . . . . . . . . . . . . . . . . . 2174

Topspin Networks and Topology in Loop Quantum Gravity

Duston, Christopher L.

- Quantum Gravity Phenomenology

Chairperson: Amelino-Camelia, Giovanni 
Quantum Gravity Effects on Laboratory Bose-Einstein

Condensates

Briscese, Fabio; Grether, Marcela; de Llano, Manuel . . . . . . . . . . 2183

Lorentz Symmetry as an Emergent Phenomenon

Consoli, Maurizio . . . . . . . . . . . . . . . . . . . . . . . . . . 2186

On the No-Gravity Limit of Gravity

Kowalski-Glikman, Jerzy; Szczachor, Michal . . . . . . . . . . . . . . 2189

MoNDian Dark Matter, Entropic Gravity, and Infinite Statistics

$\mathrm{Ng}$, Y. Jack . . . . . . . . . . . . . . . . . . . . . . . . . . . . 2192

Planck Scale Gravity Test with Accelerators

Gharibyan, Vahagn . . . . . . . . . . . . . . . . . . . . . . . . . 2195

The Quantum Clock: A Critical Discussion on Space-Time

Burderi, Luciano; Di Salvo, Tiziana . . . . . . . . . . . . . . . . . . 2203

Covariant Renormalizable Gravity Theories on

(Non) Commutative Tangent Bundles

Vacaru, Sergiu I. . . . . . . . . . . . . . . . . . . . . . . . . . . 2206

The Schrödinger-Newton Equation as a Model for Self-Gravitating

Systems

Grossardt, Andre; Giulini, Domenico . . . . . . . . . . . . . . . . . 2209

$\kappa$-Minkowski Description of Spacetime Foam: Kinematics

Astuti, Vahagn . . . . . . . . . . . . . . . . . . . . . . . . . . . 2212

Modified Particle-Reaction Kinematics from

Planck-Scale-Deformed Spacetime Symmetries

Barcaroli, Leonardo; Loret, Niccoló; Amelino-Camelia, Giovanni . . . . . 2215

Rainbow Metric Formalism and Relative Locality

Loret, Niccolò; Barcaroli, Leonardo . . . . . . . . . . . . . . . . . . 2218

Planck-Scale-Induced Spacetime Fuzziness in $\kappa$-Minkowski

Rosati, Giacomo . . . . . . . . . . . . . . . . . . . . . . . . . . 2221

- Asymptotic Safeness and Symmetry Breaking in Quantum Gravity

Chairperson: Mielke, Eckehard W.

Fixed Functionals in Asymptotically Safe Gravity

Saueressig, Frank; Demmel, Maximilian; Zanusso, Omar . . . . . . . . . 2227

Asymptotic Safety and Black Hole Thermodynamics

Becker, Daniel; Reuter, Martin . . . . . . . . . . . . . . . . . . . . 2230

Experimentally Testing Asymptotically Safe Quantum Gravity

with Photon-Photon Scattering

Eichhorn, Astrid 
The Asymptotic Safety Scenario and Scalar Field Inflation

Rahmede, Christoph . . . . . . . . . . . . . . . . . . . . 2236

IR Effects in de Sitter Spacetime: From Non-Perturbative Effects

to Gauge Artifacts

Youssef, Ahmed . . . . . . . . . . . . . . . . . . . . . . . . . . . 2239

Some Remarks on the Historical Origin and Current Prospects of Holography

Sieroka, Norman . . . . . . . . . . . . . . . . . . . . . . . . . . 2242

Asymptotic Safety of the Cartan Induced Four-Fermion

Interaction?

Mielke, Eckehard W.

- Loop Quantum Gravity: Cosmology and Black Holes

Chairperson: Pullin, Jorge; Parampreet, Singh

Loop Quantum Gravity: Cosmology and Black Holes at the 13th

Marcel Grossmann Meeting

Pullin, Jorge; Singh, Parampreet . . . . . . . . . . . . . . . . . . 2251

A Quantum Gravity Extension of the Inflationary Paradigm

Agullo, Ivan; Ashtekar, Abhay; Nelson, William . . . . . . . . . . . . . 2256

(Some) Observational Consequences of Loop Quantum Cosmology

Cailleteau, Thomas . . . . . . . . . . . . . . . . . . . . 2259

The Thermodynamic Limit for Black Holes in Loop Quantum

Gravity.

Barbero, Fernando; Villaseñor, Eduardo J.S. . . . . . . . . . . . . . 2263

Loop Quantum Cosmology for Nonminimally Coupled Scalar Field

Dapor, Andrea; Artymowski, Michal; Pawtowski, Tomasz . . . . . . . . . 2266

Geometric Time in Quantum Cosmology

Pawtowski, Tomasz . . . . . . . . . . . . . . . . . . . . . . . . . 2269

Uniqueness of the Fock Quantization of Scalar Fields With Time

Dependent Mass

Velhinho, Jose M.; Olmedo, Javier; Mena Marugán, Guillermo

A.; Cortez, Jeronimo . . . . . . . . . . . . . . . . . . . . . . . . 2272

Hybrid Quantization of an Inhomogeneous Inflationary Scenario

Fernández-Méndez, Mikel; Mena Marugán, Guillermo A.; Olmedo, Javier . 2275

Approximate Quantum Solutions in Inhomogeneous Loop

Quantum Cosmology

Martín-Benito, Mercedes; Martín-de Blas, Daniel; Mena Marugán,

Guillermo A. . . . . . . . . . . . . . . . . . . . . . . . . . . . 2278 
Quantum Field Theory on LQC Bianchi Spacetimes

Dapor, Andrea; Lewandowski, Jerzy; Tavakoli, Yaser

Inhomogeneous Universe in Loop Quantum Gravity

Cianfrani, Francesco

- Origin and Physics of Soft Gamma-ray Repeaters and Anomalous X-Ray Pulsars

Chairperson: Mereghetti, Sandro; Malheiro, Manuel

Progenitors and SNR Associated to SGR/AXPs

Horvath, Jorge E. . . . . . . . . . . . . . . . . . . . . . . . . . . 2289

The Quiescent X-Ray Emission of AXPs and SGRs - Powered by

Accretion from a Fallback Disk

Truemper, Joachim; Dennerl, Konrad; Kylafis, Nikos D.; Ertan,

Ülan: Zezas, Andreas

SGRs and AXPs as Massive Fast Rotating Highly Magnetized

White Dwards: The Case of SGR 0418+5729

Boshkayev, Kuantay; Rueda, Jorge; Ruffini, Remo . . . . . . . . . . . . 2295

Magnetic Reconnection Instabilities in Soft-Gamma Repeaters

Heyl, Jeremy S.; Gill, Ramandeep . . . . . . . . . . . . . . . . . . . 2301

Central Compact Objects in Magnetic Lethargy.

Viganò, Daniele; Pons, Jose A.; Perna, Rosalba

Constraining the Central Magnetic Field of Magnetars

Mukhopadhyay, Banibrata; Sinha, Monika

- Nuclear Physics and Astrophysics

Chairperson: Rafelski, Johann; Rueda, Jorge

Magnetism in Compact Ultra Dense Objects

Ferrer, Efrain J.

Magnetized Compact Stars

Pérez Martínez, Aurora; González, Felipe Ricardo; Mareza Paret, Daryel . . 2316

Nuclear Symmetry Energy and New Degrees of Freedom at Higher

Density in Compact Stars

Lee, Hyun Kyu

Astrophysical Signatures of Quark Stars in the CFL (Color-Flavor Locked) Phase

Harko, Tiberiu

- Strong Fields and High Energy Astrophysical Events

Chairperson: Ghezzi, Cristián; Xue, She-Sheng

Session Summary: Strong Fields and High Energy Astrophysical

Events 
Xue, She-Sheng . . . . . . . . . . . . . . . . . . . . . . . . . . 2327

The Wrapping of Magnetic Field Lines Due to Frame Dragging

Around a Neutron Star

Qadir, Asghar; Herbst, Rhameez S.; Momoniat, Ebrahim . . . . . . . . . 2330

The Solution of Thomas-Fermi Equation in the Presence of the

Strong Magnetic Fields

Mohammadi, Rohoollah; Xue, She-Sheng . . . . . . . . . . . . . . . . 2334

Electron and Positron Pair Production in Gravitational Collapses

Xue, She-Sheng . . . . . . . . . . . . . . . . . . . . . . . . . . 2338

Oscillatory Motion and Acceleration of String Loops in the Field of Black Hole

Kološ, Martin; Stuchlık, Zdenk . . . . . . . . . . . . . . . . . 2341

Numerical Study of Relativistic Magnetohydrodynamic

Reconnection and Its Radiative Effects

Takahashi, Hiroyuki R.; Ohsuga, Ken . . . . . . . . . . . . . . . . . 2344

On the Stability of Circular Orbits in Galactic Dynamics:

Newtonian thin Disks

Vieira, Ronaldo S.S.; Ramos-Caro, Javier . . . . . . . . . . . . . . . 2346

- Relativistic MHD Flows and Electron-Positron Plasma

Chairperson: Lou, Yu-Qing

A Model of Torus Structure of Vela Pulsar Wind Nebula. Determination of the Leptonic Distribution Function Anisotropy.

Uvarov, Iurii A.; Bykov, Andrei M.

- GR around the Earth and the Sun

Chairperson: Peron, Roberto; Ashby, Neil

Simulations of Solar System Observations in Alternative Theories of Gravity

Hees, Aurelien; Lamine, B.; Reynaud, S.; Jaekel, M.-T.; Le

Poncin-Lafitte, C.; Lainey, V.; Füzfa, A.; Courty, J.-M.; Dehant, V.; Wolf, P.

GINGER: Measuring Gravitomagnetic Effects By Means of Light Tartaglia, Angelo . . . . . . . . . . . . . . . . . . . . . . . . . . 2360

Torsion of the Earth's Anomalous Gravitational Field Resulting from the Finite Speed of the Gravitational Interaction Zielinski, Janusz B. . . . . . . . . . . . . . . . . . . . . . . . . . 2363

Venus Transits: History and Opportunities for Planetary, Solar and Gravitational Physics

Sigismondi, Costantino; Wang, Xiaofan; Rocher, Patrick; Reis Neto, Eugenio 
GAME: A Space Mission for Relativistic Tests Around the Earth

Vecchiato, Alberto; Gai, Mario; Lattanzi, Mario G.; Ligori, S.

Relativistic Positioning, Pulsars and Space-Time Geodesy

Tartaglia, Angelo . . . . . . . . . . . . . . . . . . . . . . . . . . 2375

- Multi-Dimensional Codes for Supernova Explosions

Chairperson: Arnett, David; Meakin, Casey

Computations of the Gravitational Collapse of a Stellar Core with

Neutrinos Transport and the Problem of Supernovae

Aksenov, Alexey G.; Chechetkin, Valery M. . . . . . . . . . . . . . . . 2381

- Supernova Explosions and Neutron Star Oscillations

Chairperson: Kokkotas, Konstantinos; Mueller, Ewald

Distortion of Neutron Stars with a Toroidal Magnetic Field

Frieben, Joachim; Rezzolla, Luciano . . . . . . . . . . . . . . . . . . 2387

The $f$-Mode Instability in Relativistic Neutron Stars

Gaertig, Erich; Kokkotas, Kostas D. . . . . . . . . . . . . . . . . . . 2390

- Spectral and Timing Properties of Astrophysical Black Holes

Chairperson: Chakrabarti, Sandip

Model of a Disk-Outflow Coupled System: Disk-Outflow Symbiosis

Mukhopadhyay, Banibrata . . . . . . . . . . . . . . . . . . . . . . 2395

The Accretion-Ejection Instability in Microquasars

Varniere, Peggy; Tagger, Michel; Rodriguez, Jerôme . . . . . . . . . . . 2398

Numerical Simulations of a Two Component Advective Flow for

the Study of the Spectral and Timing Properties of Black Holes

Giri, Kinsuk; Chakrabarti, Sandip Kumar . . . . . . . . . . . . . . . 2401

Effects of Compton Cooling on Outflow in a Two Component

Accretion Flow Around a Black Hole: Results of Coupled Monte

Carlo-TVD Simulation

Garain, Sudip K; Ghosh, Himadri; Chakrabarti, Sandip K . . . . . . . . 2404

A Possible Origin for the HFQPOs in Microquasars

Varniere, Peggy; Tagger, Michel; Vincent, Frédéric; Meheut, Heloise . . . 2407

A Comparative Study of the Timing and the Spectral Properties

During Two Recent Outbursts (2010 and 2011) of H 1743-322

Debnath, Dipak; Chakrabarti, Sandip K.; Nandi, Anuj . . . . . . . . . . 2410

A Variability Based Classification for Microquasar

Varniere, Peggy; Tagger, Michel; Rodriguez, Jerôme . . . . . . . . . . . 2412

Testing Strong Gravity with Spectral and Temporal Behavior of

Some Seyfert Nuclei

Tsuruta, Sachiko 
Low Angular Momentum Flow Model for Sgr A*

Okuda, Toru; Molteni, Diego . . . . . . . . . . . . . . . . . . . . . 2418

A Possible Origin for the LFQPO Types in Microquasars

Varniere, Peggy; Tagger, Michel; Rodriguez, Jerôme . . . . . . . . . . . 2421

Properties of the Propagating Oscillatory Shock Wave in the

Accretion Flows Around Few Transient Black Hole Candidates

during Their Outbursts

Debnath, Dipak; Chakrabarti, Sandip K

- Observations from High Energy Astrophysics Satellites

Chairperson: Schulz, Norbert S.; Pian, Elena

The Mystery of the $\gamma$ Cas Type X-Ray Sources

Torrejon, Jose M. . . . . . . . . . . . . . . . . . . . . . . . . . . 2429

The High Energy View of the Globular Cluster NGC 6388: Is an

Intermediate Mass Black Hole Out There?

Nucita, Achille A.; de Paolis, Francesco; Saxton, Richard; Read, Andrew M.; Ingrosso, Gabriele; Vetrugno, Daniele; Manni, Luigi . . . . . 2432

- White Dwarf Pulsars and Rotating White Dwarf Theory

Chairperson: Terada, Yukikatsu

High-Energy Observations in the Search for Non-Thermal

Emissions from Accelerated Particles in Magnietic

White Dwarfs: A Review

Terada, Yukikatsu . . . . . . . . . . . . . . . . . . . . . . . . . 2437

Suzaku Searches for WD Pulsars with a Spectral Model of

Post-Shock Regions

Hayashi, Takayuki; Terada, Yukikatsu; Harayama, Atsushi; Ishida, Manabu 2440

Preliminary Hard X-Ray Results of AE Aquarii Observed with

NuSTAR

Kitaguchi, Takao; An, Hongjun; Rana, Vikram R.; Kaspi, Victoria

N.; Gotthelf, Eric V.; Harrison, Fiona A.; Boggs, Steven E.;

Christensen, Finn E.; Craig, William W.; Hailey, Charles J.;

Stern, Daniel; Zhang, Will W.

Search for Non-Thermal Emission from Isolated Magnetized White

Dwarfs

Harayama, Atsushi; Terada, Yukikatsu; Hayashi, Takayuki; Ishida, Manabu 2447

Particle Simulation for Rotating White Dwarf Magnetopshere

Wada, Tomohide; Fujisawa, Kotaro . . . . . . . . . . . . . . . . . . 2450

Formation and Appearance of Pulsar-Like White Dwarfs

Ikhsanov, Nazar R.; Beskrovnaya, Nina G. . . . . . . . . . . . . . . . 2453

The X-Ray Source 1RXS J180431.1-273932: The Importance of 
Being Pinpointed

Nucita, Achille A.; Masetti, Nicola; Parisi, Pietro . . . . . . . . . . . . 2456

RX J0648.0-4418: The Fastest-Spinning White Dwarf

Mereghetti, Sandro . . . . . . . . . . . . . . . . . . . . . . . . . 2459

Magnetic Fields of SGRs/AXPs as Rotation-Powered Massive

White Dwarf Pulsars

Malheiro, Manuel; Coelho, Jaziel Goulart . . . . . . . . . . . . . . . 2462

Do SGRs/AXPs and Radio AXPs Have the Same Nature?

Coelho, Jaziel Goulart; Malheiro, Manuel . . . . . . . . . . . . . . . 2465

General Relativistic and Newtonian White Dwarfs

Boshkayev, Kuantay; Rueda, Jorge; Ruffini, Remo; Siutsou, Ivan . . . . . 2468

On the Analog Gravity Formalism Applied to White Dwarfs

Filippi, Simonetta; Cherubini, Christian; Bini, Donato . . . . . . . . . . 2475

Strongly Magnetized Electron Degenerate Gas: Highly

Super-Chandrasekhar White Dwarfs

Das, Upasana; Mukhopadhyay, Banibrata . . . . . . . . . . . . . . . 2478

On the Relativistic Feynman-Metropolis-Teller Equation of State

at Finite Temperatures

Martins de Carvalho, Sheyse; Rueda, Jorge A.; Ruffini, Remo . . . . . . 2481

- Future Experiments and Missions in X and Gamma Ray

Chairperson: Frontera, Filippo; Zhang, Shuang-Nan

Status and Prospects of the X-Ray Astronomy Satellite ASTRO-H

Tsujimoto, Masahiro; Liu, J. . . . . . . . . . . . . . . . . . . . . . 2487

LOFT - The Large Observatory for X-Ray Timing

Bozzo, Enrico; Esposito, Paolo; Romano, Patrizia . . . . . . . . . . . . 2490

- Planckian and Transplanckian Physics

Chairperson: Gal'tsov, Dmitry

Production and Evaporation of Planck Scale Black Holes at the

LHC

Nicolini, Piero; Mureika, Jonas; Spallucci, Euro; Winstanley,

Elizabeth; Bleicher, Marcus . . . . . . . . . . . . . . . . . . . . . . 2495

Finite Zero Point Gravitational Energy in the Context of Modified

Dispersion Relations

Garattini, Remo; Mandanici, Gianluca . . . . . . . . . . . . . . . . . 2498

Plasmon-Graviton Conversion in a Magnetic Field in TeV-Scale

Gravity

Melkumova, Elena 
Transplanckian Gravitational Bremsstrahlung in Post-Linear

Formalism

Gal'tsov, Dmitry; Spirin, Pavel . . . . . . . . . . . . . . . . . . . . 2504

Ultrarelativistic Brane Collisions

Gal'tsov, Dmitry; Melkumova, Elena; Spirin, Pavel . . . . . . . . . . . 2507

- String Theory

Chairperson: Henningson, Måns

Friedel Oscillations and Holographic Liquids

Puletti, Valentina Giangreco Marotta . . . . . . . . . . . . . . . . . 2513

New Massive Gravity on the de Sitter Space and Black Holes at

the Special Point

Gabadadze, Gregory; Giribet, Gaston; Iglesias, Alberto . . . . . . . . . . 2516

String-Like Structures in the Real and Complex Kerr Geometry

Burinskii, Alexander . . . . . . . . . . . . . . . . . . . . . . . . 2519

Fuzzy Objects and Noncommutative Solitons

Kobayashi, Shinpei; Asakawa, Tsuguhiko . . . . . . . . . . . . . . . . 2522

- Multivalued Fields for Defects in Spacetime and Gravity

Chairperson: Kleinert, Hagen; Katanaev, Mikhail

Multivalued Fields for Defects in Spacetime and Gravity

Kleinert, Hagen; Katanaev, Mikhail . . . . . . . . . . . . . . . . . . 2527

Multivalued Fields and Einstein-Cartan Geometry

Kleinert, Hagen . . . . . . . . . . . . . . . . . . . . . . . . . . . 2530

Some Aspects of Defect Theory in Spacetime

Kleman, Maurice . . . . . . . . . . . . . . . . . . . . . . . . . . 2531

The Einsteinian Translational Gauging and the Dislocations With

Finite-Sized Core

Malyshev, Cyril . . . . . . . . . . . . . . . . . . . . . . . . . . . 2534

Cylindrical Geometric Defects and Multi-Wall Nanotubes

Konstantinova, Elena; Katanaev, Mikhail O.; De Berredo-Peixoto,

Guilherme; Shapiro, Ilya L. . . . . . . . . . . . . . . . . . . . . . 2537

- Inhomogeneous Cosmologies, Averaging and Back Reaction

Chairperson: Coley, Alan; Wiltshire, David

Fully Characterizing Axially Symmetric Szekeres Models with

Three Data Sets

Celerier, Marie-Noëlle; Mishra, Priti; Singh, Tejinder P. . . . . . . . . . 2541

Evolution of Density Perturbations in Large Void Universe

Nishikawa, Ryusuke; Yoo, Chul-Moon; Nakao, Ken-ichi . . . . . . . . . 2544

Perturbations of Kantowski-Sachs Models 
Bradley, Michael; Forsberg, Mats; Keresztes, Zoltán; Gergely,

László Á.; Dunsby, Peter K.S. . . . . . . . . . . . . . . . . . . . . 2547

Homogenization of Scalar Field Cosmologies

Mena, Filipe; Alho, Artur . . . . . . . . . . . . . . . . . . . . . . 2550

Back-Reaction in Relativistic Cosmology

Clifton, Timothy . . . . . . . . . . . . . . . . . . . . . . 2553

Apparent Acceleration and an Alternative Concordance from

Causal Backreaction

Bochner, Brett .......................... 2566

Modeling the Past Null Cone Using Characteristic Numerical

Relativity

van der Walt, Petrus J. ; Bishop, Nigel T. . . . . . . . . . . . . . . . 2569

A Lattice Universe as a Toy Model for Inhomogeneous Cosmology

Bruneton, Jean-Philippe . . . . . . . . . . . . . . . . . . . . . . . 2572

Black Hole Universe

Yoo, Chul-Moon; Abe, Hiroyuki; Nakao, Ken-ichi; Takamori, Yohsuke . . . 2575

Averaging via Cartan Scalars

Kašpar, Petr; Svitek, Otakar . . . . . . . . . . . . . . . . . . . . . 2578

SUSY and Dark Matter Results from ATLAS

Sandaker, Heidi; ATLAS-collaboration . . . . . . . . . . . . . . . . 2581

List of Participants . . . . . . . . . . . . . . . . . . . . . . . . . 2587

Author Index . . . . . . . . . . . . . . . . . . . . . . . . 2609 